\title{
CORRIGENDUM
}

\section{Simultaneous measurement of nanoscale electric and magnetic optical fields}

B. le Feber, N. Rotenberg, D. M. Beggs and L. Kuipers

Nature Photonics 8, 43-46 (2014); published online: 15 December 2013; corrected after print: 30 January 2014

The reference section in the print version of this Letter contained the following errors:

For ref. 3, the volume number should have been 4 rather than 3 .

For ref. 17, “15, 1289-1295" should have been "http://dx.doi.org/10.1126/science.1232009”.

For ref. 30, the volume number should have been 326 rather than 23 .

The online HTML and PDF versions of the Letter do not contain these errors. 